Pacific Journal of Mathematics

MATRIX REPRESENTATIONS FOR LINEAR
TRANSFORMATIONS ON ANALYTIC SEQUENCES 


\title{
MATRIX REPRESENTATIONS FOR LINEAR TRANSFORMATIONS ON ANALYTIC SEQUENCES
}

\author{
Philip C. Tonne
}

\begin{abstract}
Let $\mathscr{A}$ be the space of all analytic sequences, those complex sequences $\alpha$ for which there is a positive number $r$ such that $\sum \alpha_{n} r^{n}$ converges. Those linear transformations from $\mathscr{A}$ to $\mathscr{A}$ which have matrix representations are characterized in terms of various spaces and topologies associated with $\mathscr{A}$. An example is given of a linear transformation from $\mathscr{A}$ to $\mathscr{A}$ which has no matrix representation.
\end{abstract}

Louise Raphael [8] characterizes the matrix transformations from $\mathscr{A}$ to $\mathscr{A}$. She makes use of the following: if $q>0, A_{q}$ is the subspace of $\mathscr{A}$ to which $\alpha$ belongs only in case $\left\{\left|\alpha_{n}\right| q^{n}\right\}_{n=0}^{\infty}$ is a bounded sequence, and $\|\alpha\|_{q}$ denotes the least number less than no term of that bounded sequence. If $q>0,\left\{A_{q},\|\|_{q}\right\}$ is a complete normed linear space. (See also, I. Heller [5], I. M. Sheffer [10, Th. 6, p. 177], and the more fundamental work of Karl Zeller [12].)

Following M. G. Haplanov [4] and V. Ganapathy Iyer [3], $S_{q}$ denotes the subset of $\mathscr{A}$ to which $\alpha$ belongs provided that $\sum \alpha_{n} z^{n}$ converges for $|z|<q$, and, if $0<p<q, N_{p}(\alpha)$ denotes $\sum_{k=0}^{\infty}\left|\alpha_{k}\right| p^{k}$ for each $\alpha$ in $S_{q}$. If $q>p>0,\left\{S_{q}, N_{p}\right\}$ is a normed linear space (not complete).

In [11] the author characterizes those linear transformations from $S_{1}$ to $S_{1}$ which have matrix representations. We continue here in much the same spirit. If $q>0$ and $\alpha=\left\{\alpha_{n}\right\}_{n=0}^{\infty}$ is a sequence of sequences in $\mathscr{A}$ and $f$ is a sequence of analytic functions such that if $n$ is a nonnegative integer and $|z|<q$ then

$$
f_{n}(z)=\sum_{k=0}^{\infty} \alpha_{n k} z^{k}
$$

and $f$ converges uniformly with limit 0 on each closed subset of the (open) disc with center 0 and radius $q$, then $\alpha$ is said to have limit 0 analytically relative to $q$. A sequence has limit 0 analytically if it has limit 0 analytically relative to some positive number.

We recall some fundamental notions from G. Köthe and $O$. Toeplitz [7] about sequence spaces:

Suppose that $\lambda$ is a linear sequence space. $\lambda^{*}$ (sometimes called the dual or $\alpha$-dual of $\lambda$ ) is the collection of all complex sequences $y$ such that $\sum\left|y_{n} x_{n}\right|$ converges for each $x$ in $\lambda$. If $x$ is in $\lambda$ and $y$ is in $\lambda^{*}$, 


$$
Q(x, y)=\sum_{n=0}^{\infty} x_{n} y_{n}
$$

A sequence $x=\left\{x_{p}\right\}_{p=0}^{\infty}$ of sequences in $\lambda$ is said to converge in $\lambda$ provided that, for each $y$ in $\lambda^{*}$, the complex sequence $\left\{Q\left(x_{p}, y\right)\right\}_{p=0}^{\infty}$ converges. The transformation $F$ is sequentially continuous from $\lambda$ to $\lambda$ provided that $\left\{F\left(x_{p}\right)\right\}_{p=0}^{\infty}$ converges in $\lambda$ if $\left\{x_{p}\right\}_{0}^{\infty}$ converges in $\lambda$.

Theorems $\mathrm{A}$ and $\mathrm{B}$ are due to Köthe and Toeplitz.

THEOREM A. If $\lambda=\lambda^{* *}$ and the matrix $M$ transforms $\lambda$ to $\lambda$ (if $x$ is in $\lambda$ and $y_{n}=\sum_{k=0}^{\infty} M_{n k} x_{k}, n=0,1, \cdots$, then $y$ is in $\lambda$ ), then the transformation is sequentially continuous from $\lambda$ to $\lambda$ [7, Satz 6, p. 206].

THEOREM B. Each linear sequentially-continuous transformation from $\lambda$ to $\lambda$ has a matrix representation. [7, Satz 7, p. 207].

A subset $X$ of the sequence space $\lambda$ is bounded in $\lambda$ if for each $u$ in $\lambda^{*}$ there is a number $m$ such that if $x$ is in $X$ then $|Q(x, u)| \leqq$ $m$. If $F$ is a transformation from $\lambda$ to $\lambda$, the adjoint $F^{*}$ of $F$ is the relation to which the ordered pair $\{x, y\}$ belongs only in the case that

$$
Q(x, F(z))=Q(y, z)
$$

for each $z$ in $\lambda$.

Let $\mathscr{E}$ be the space of all entire sequences, those complex sequences which are coefficient sequences for power-series expansions of entire functions. $\mathscr{E}=\mathscr{A}^{*}$ and $\mathscr{E}^{*}=\mathscr{A}$. The matrix transformations from $\mathscr{E}$ to $\mathscr{E}$ have been characterized by H. I. Brown [1] and, in another manner, by K. Chandrasekhara Rao [2].

Theorem. Let $L$ be a linear transformation from $\mathscr{A}$ to $\mathscr{A}$. These statements are equivalent:

(1) L has a matrix representation.

(2) $L$ is sequentially continuous from $\mathscr{A}$ to $\mathscr{A}$.

(3) If $p>0$ there is a positive number $q$ such that $L$ maps $\left\{A_{p},\|\|_{p}\right\}$ continuously into $\left\{A_{q},\|\|_{q}\right\}$ (with respect to the norms).

( $\left.3^{\prime}\right)$ If $p>0$ there is a positive number $q$ such that $L$ maps $A_{p}$ into $A_{q}$.

(4) If $X$ is a set bounded in $\mathscr{A}$ then $L(X)$ is also.

(5) If $0<p<r$ there is a positive number $R$ such that, if $0<P<R$, then $L$ maps $\left\{S_{r}, N_{p}\right\}$ into $\left\{S_{R}, N_{P}\right\}$ continuously.

(6) $L^{*}$ is a sequentially continuous transformations from $\mathscr{E}$ to $\mathscr{E}$.

(7) If $\alpha$ has limit 0 analytically, so does $\left\{L\left(\alpha_{n}\right)\right\}_{n=0}^{\infty}$. 
$\mathscr{A}^{* *}=\mathscr{A}$ and $\mathscr{E}^{* *}=\mathscr{E}$. This and the following lemmas are useful in the proof of our theorem.

Lemma 0. Suppose that $\lambda$ is a sequence space and $\lambda^{* *}=\lambda$ and $T$ is a linear sequentially continuous transformation from $\lambda$ to $\lambda$. Then $T^{*}$ is a sequentially continuous transformation from $\lambda^{*}$ into $\lambda^{*}$.

Via [7, Satz 6, p. 200], a characterization of linear functionals, Lemma 0 is easy to prove. (See also [9, p. 158].)

Lemma 1. If $B$ is a set bounded in $\mathscr{A}$, then there is a member $\alpha$ of $\mathscr{A}$ such that if $\beta$ is in $B$ then $\left|\beta_{k}\right| \leqq \alpha_{k}, k=0,1, \cdots$.

Proof. Otherwise, there is a sequence $\beta$ of sequences in $B$ and an increasing sequence $n$ of nonnegative integers such that, if $k$ is a positive integer, $\left|\beta_{k, n_{k}}\right|>k^{1+n_{k}}$. Let us indicate how to define such a sequence. Let $\beta_{1}$ be a member of $B$ and $n_{1}$ be a positive integer such that $\left|\beta_{1, n_{1}}\right|>1^{1+n_{1}}$. Let $t$ be a number such that if $b$ is in $B$ then $\left|b_{k}\right| \leqq t, k=0,1, \cdots, n_{1}$. Let $\beta_{2}$ be a member of $B$ and $n_{2}$ be a positive integer such that $\left|\beta_{2, n_{2}}\right|>t \cdot 2^{1+n_{2}} \cdot n_{2}>n_{1}$. Please continue.

Let $e$ be a sequence such that if $k$ is a nonnegative integer then $e_{n_{k}}=k^{-n_{k}}$ and $e_{k}=0$ if there is no positive integer $j$ such that $n_{j}=k . e$ is in $\mathscr{E}$.

The set $D$ to which $d$ belongs only in case $\left|d_{k}\right| \leqq\left|e_{k}\right|, k=$ $0,1, \cdots$, is bounded in $\mathscr{E}$. Since $B$ is bounded, it is strongly bounded (see [7, Satz 1, p. 201] or [6, p. 413 (5)], so that there is a number $c$ such that if $b$ is in $B$ and $d$ is in $D$ then $|Q(b, d)| \leqq c$. Let $k$ be a positive integer. Let $u$ be a complex sequence such that if $j$ is a nonnegative integer then $\left|u_{j}\right|=1$ and $\beta_{k j} u_{j} \geqq 0 . u \cdot e$ is in $D$.

$$
\begin{aligned}
c & \geqq\left|Q\left(\beta_{k}, u \cdot e\right)\right|=\left|\sum_{j=0}^{\infty} \beta_{k j} u_{j} e_{j}\right|=\sum_{j=0}^{\infty} \beta_{k j} u_{j} e_{j} \\
& \geqq \beta_{k, n_{k}} u_{n_{k}} e_{n_{k}}=\left|\beta_{k, n_{k}}\right| e_{n_{k}}>k^{1+n_{k}} k^{-n_{k}}=k .
\end{aligned}
$$

So there is a member $\alpha$ of $\mathscr{A}$ such that if $b$ is in $B$ then $\left|b_{k}\right| \leqq \alpha_{k}, k=0,1, \cdots$.

Lemma 2. If $\alpha$ is a sequence of sequences in $\mathscr{A}$, then these are equivalent:

(1) $\alpha$ has limit 0 analytically.

(2) $\alpha$ has limit 0 in $\mathscr{A}$.

Proof. Suppose that $\alpha$ has limit 0 analytically (relative to $q$ ). Then $\alpha$ has limit 0 in $S_{q}$ (see [11, Lemma 1]). $\alpha$ is a sequence 
bounded in $S_{q} \cdot \mathscr{A}^{*}$ is a subset of $S_{q}^{*}$, so $\alpha$ is a sequence bounded in $\mathscr{A}$, and there is a member $\beta$ of $\mathscr{A}$ such that if each of $j$ and $k$ is a nonnegative integer then $\left|\alpha_{j k}\right| \leqq \beta_{k}$. Let $t$ be a positive number such that $\beta_{k} \leqq t^{k+1}, k=0,1, \cdots$. Let $e$ be in $\mathscr{E} .\left(\mathscr{E}=\mathscr{A}^{*}.\right)$ Let $\varepsilon$ be a positive number. Let $m$ be a positive integer such that $2 \sum_{k=m}^{\infty}\left|e_{k}\right| t^{k+1}<\varepsilon$. Let $J$ be a positive interger such that if $j$ is an integer exceeding $J$ then $2 \sum_{k=0}^{m-1}\left|a_{j k}\right|\left|e_{k}\right|<\varepsilon$. Then, if $j>J$,

$$
\begin{aligned}
\left|Q\left(\alpha_{j}, e\right)\right| & =\left|\sum_{k=0}^{\infty} \alpha_{j k} e_{k}\right| \leqq \sum_{k=0}^{\infty}\left|\alpha_{j k}\right|\left|e_{k}\right| \\
& \leqq \sum_{k=0}^{m-1}\left|\alpha_{j k}\right|\left|e_{k}\right|+\sum_{k=m}^{\infty}\left|e_{k}\right| t^{k+1}<\varepsilon .
\end{aligned}
$$

So $\alpha$ has limit 0 in $\mathscr{A}$.

Now, suppose that $\alpha$ has limit 0 in $\mathscr{A} \cdot \alpha$ is a sequence bounded in $\mathscr{A}$. There is a positive number $t$ such that $\left|\alpha_{j k}\right| \leqq t^{k+1}, j, k=$ $0,1, \cdots$. Let $q$ be a number between 0 and $1 / t$. Let $\varepsilon$ be a positive number. Let $m$ be a positive integer such that $2 \sum_{k=m}^{\infty} q^{k} t^{k+1}<$ $\varepsilon$. Let $J$ be a positive integer such that if $j$ is an integer exceeding $J$ then $2 \sum_{k=0}^{m-1}\left|\alpha_{j k}\right| q^{k}<\varepsilon$. Now, if $j>J$ and $|z| \leqq q$,

$$
\left|\sum_{k=0}^{\infty} \alpha_{j k} z^{k}\right| \leqq \sum_{k=0}^{\infty}\left|\alpha_{j k}\right| q^{k} \leqq \varepsilon
$$

So $\alpha$ has limit 0 analytically relative to $1 / t$.

Lemma 3. Suppose that $r>p>0$ and $R>P>0$ and $L$ is a continuous linear transformation from $\left\{S_{r}, N_{p}\right\}$ to $\left\{S_{R}, N_{P}\right\}$. Then $L$ has a matrix representation.

Proof. By [11, Theorem 1] this is true if $r=R=1$.

Suppose that, for each positive number $\rho, t(\rho)$ is the function from $\mathscr{A}$ to $\mathscr{A}$ such that if $\alpha$ is in $\mathscr{A}$ and $n$ is a nonnegative integer then $t(\rho)(\alpha)_{n}=\alpha_{n} \rho^{n}$, so that, if $0<q<\rho, t(\rho) \operatorname{maps}\left\{S_{\rho}, N_{q}\right\}$ continuously onto $\left\{S_{1}, N_{q / \rho}\right\}$.

Let $L^{\prime}$ be the continuous linear transformation from $\left\{S_{1}, N_{p l r}\right\}$ into $\left\{S_{1}, N_{P / R}\right\}$ such that if $x$ is in $S_{1}$ then

$$
L^{\prime}(x)=t(R) L t(1 / r)(x) \text {. }
$$

$L^{\prime}$ has a matrix representation, so $L$ has a matrix representation.

Lemma 4. Suppose that $0<p<r$. If $\alpha$ is in $A_{r}$, then $\alpha$ is in $S_{r}$ and

$$
N_{p}(\alpha) \leqq\|\alpha\|_{r} /(1-p / r) \text {. }
$$


If $\alpha$ is in $S_{r}$, then $\alpha$ is in $A_{p}$ and

$$
\|\alpha\|_{p} \leqq N_{p}(\alpha) \text {. }
$$

The proof is straight-forward and omitted.

Proof of Theorem. 1↔2. That statements (1) and (2) are equivalent is seen from Theorems $\mathrm{A}$ and $\mathrm{B}$.

$1 \rightarrow 3$. Mrs. Raphael has shown that statement (3) follows from (1) [8, Theorem 4, p. 124].

$2 \rightarrow 4$. That statement (4) follows from (2) is a consequence of [7, Satz 5, p. 207].

$4 \rightarrow 3^{\prime}$. Suppose that if $X$ is a set bounded in $\mathscr{A}$ then $L(X)$ is too. Let $p$ be a positive number. Let $X$ be the set of all points $x$ of $A_{p}$ such that $\|x\|_{p} \leqq 1$. Let $e$ be in $E$. Let $x$ be in $X$.

$$
|Q(x, e)|=\left|\sum_{k=0}^{\infty} e_{k} x_{k}\right| \leqq \sum_{k=0}^{\infty}\left|e_{k}\right|\left|x_{k}\right| \leqq \sum_{k=0}^{\infty}\left|e_{k}\right| p^{-k},
$$

so $X$ is bounded in $A$.

$L(X)$ is bounded in $A$. By Lemma 1 there is a positive number $q$ such that if $y$ is in $L(X)$ then $\left|y_{n}\right| \leqq q^{n+1}, \mathrm{n}=0,1, \ldots$. So, if $x$ is in $A_{p}, L(x)$ is in $A_{q}$. Therefore statement ( $\left.3^{\prime}\right)$ follows from statement (4).

$3^{\prime} \rightarrow 1$. That statement $3^{\prime}$ implies that statement $(1)$ is true is evident from part 4 of Karl Zeller's theorem in [12].

$2 \leftrightarrow 6$. That statements (2) and (6) are equivalent is a consequence of Lemma 0. One might also use Theorems A and B (of [7]) and [7, Satz 4, p. 206].

$2 \leftrightarrow 7$. That statements (2) and (7) are equivalent is evident from Lemma 2.

$3 \rightarrow 5$. Suppose that $0<p<r$. Let $q$ be a positive number such that $L$ maps $\left\{A_{p},\|\|_{p}\right\}$ continuously into $\left\{A_{q},\|\|_{q}\right\}$. Let $K$ be a positive number such that if $x$ is in $A_{p}$ then $\|L(x)\|_{q} \leqq K\|x\|_{p}$. Let $P$ be a number between 0 and $q$. Then, by Lemma 4, if $x$ is in $S_{r}, x$ is in $A_{p}, L(x)$ in $A_{q}, L(x)$ is in $S_{q}$, and

$$
N_{p}(L(x)) \leqq \frac{\|L(x)\|_{q}}{1-P / q} \leqq \frac{K}{1-P / q}\|x\|_{p} \leqq \frac{K}{1-P / q} N_{p}(x) .
$$

So statement (5) follows from statement (3).

$5 \rightarrow 1$. Since each point of $A$ belongs to $S_{r}$ for some positive number $r$, it follows from Lemma 3 that $L$ has a matrix representation (statement (1)) if statement (5) is true.

One can add to the seven statements in the theorem by taking other combinations of these spaces and notions. I have presented those which seem most interesting. 
ExAMPLE. Let $S$ be a maximal linearly independent subset of $A$ which contains the unit vectors $(1,0,0, \cdots)$, etc., and the constant sequence $k=(1,1, \cdots)$. We define a function $l$ from $S$ to the plane such that if $s$ is in $S$ and $s \neq k$ then $l(s)=0$ and $l(k)=1$. Let $l^{\prime}$ be the linear extension of $l$ to $A$. Let $L$ be the linear transformation from $A$ to $A$ such that if $x$ is in $A$ and $n$ is a nonnegative integer then

$$
L(x)_{n}=l^{\prime}(x) .
$$

$L$ is a linear transformation from $A$ to $A$ (indeed to the constant sequences) and, since $l^{\prime}$ cannot be represented by a sequence, $L$ has no matrix representation.

The author is indebted to Albert Wilansky for his encouragement on this project.

\section{REFERENCES}

1. H. I. Brown, Entire methods of summation, Compositio Math., 21 (1969), 35-42.

2. K. Chandrasekhara Rao, Matrix transformations on some sequence spaces, Pacific J. Math., 31 (1969), 171-174.

3. V. Ganapathy Iyer, On the space of integral functions, III, Proc. Amer. Math. Soc., 3 (1952), 874-883.

4. M. G. Haplanov, Linear transformations of analytic spaces, Amer. Math. Soc., Trans., (2) 13 (1960), 177-183.

5. I. Heller, Contributions to the theory of divergent series, Pacific J. Math., 2 (1952), 153-177.

6. Gottfried Köthe, Topological Vector Spaces I, Springer-Verlag, 1969.

7. G. Köthe and O. Toplitz, Lineare Räume mit unendlichen Koordinaten und Ringe unendlicher Matrizen, Journal für die reine und angewandte Mathematik, 171 (1934), 193-226.

8. Louise A. Raphael, On a characterization of infinite complex matrices mapping the space of analytic sequences into itself, Pacific J. Math., 27 (1968), 123-126.

9. Helmut H. Schaefer, Topological Vector Spaces, Springer-Verlag, New York, 1971.

10. I. M. Sheffer, Systems of linear equations of analytic type, Duke Math. J., 11 (1944), 167-180.

11. Philip C. Tonne, Matrix representations for linear transformations on series analytic in the unit disc, Pacific J. Math., (to appear).

12. Karl Zeller, Transformationen des Durchschnitts und der Vereinigung von Folgenräugen, Math. Nachrichten, 10 (1953) 175-177.

Received January 6, 1972.

EMORY UNIVERSITY 


\section{PACIFIC JOURNAL OF MATHEMATICS}

\section{EDITORS}

D. Gilbarg and J. Milgram Stanford University

Stanford, California 94305

R. A. Beaumont

University of Washington

Seattle, Washington 98105
J. DugundJI

Department of Mathematics

University of Southern California

Los Angeles, California 90007

RICHARD ARENS

University of California

Los Angeles, California 90024

ASSOCIATE EDITORS
E. F. BECKENBACH
B. H. NeumanN
F. WOLF
K. YoSHIDA

\section{SUPPORTING INSTITUTIONS}

UNIVERSITY OF BRITISH COLUMBIA

CALIFORNIA INSTITUTE OF TECHNOLOGY

UNIVERSITY OF CALIFORNIA

MONTANA STATE UNIVERSITY

UNIVERSITY OF NEVADA

NEW MEXICO STATE UNIVERSITY

OREGON STATE UNIVERSITY

UNIVERSITY OF OREGON

OSAKA UNIVERSITY
UNIVERSITY OF SOUTHERN CALIFORNIA

STANFORD UNIVERSITY

UNIVERSITY OF TOKYO

UNIVERSITY OF UTAH

WASHINGTON STATE UNIVERSITY

UNIVERSITY OF WASHINGTON

$\stackrel{*}{*} \stackrel{*}{*} \stackrel{*}{*}$ AMERICAN MATHEMATICAL SOCIETY

NAVAL WEAPONS CENTER 


\section{Pacific Journal of Mathematics}

\section{Vol. 46, No. $1 \quad$ November, 1973}

Allan Francis Abrahamse, Uniform integrability of derivatives on $\sigma$-lattices .......................................... 1

Ronald Alter and K. K. Kubota, The diophantine equation $x^{2}+D=p^{n} \ldots \ldots \quad 11$

Grahame Bennett, Some inclusion theorems for sequence spaces .......... 17

William Cutler, On extending isotopies ........................ 31

Robert Jay Daverman, Factored codimension one cells in Euclidean

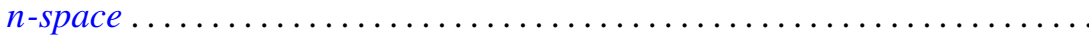

Patrick Barry Eberlein and Barrett O’Neill, Visibility manifolds ............ 45

M. Edelstein, Concerning dentability .......................... 111

Edward Graham Evans, Jr., Krull-Schmidt and cancellation over local

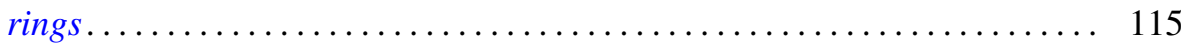

C. D. Feustel, A generalization of Kneser's conjecture ................ 123

Avner Friedman, Uniqueness for the Cauchy problem for degenerate parabolic equations .......................................... 131

David Golber, The cohomological description of a torus action ............ 149

Alain Goullet de Rugy, Un théorème du genre "Andô-Edwards" pour les

Fréchet ordonnés normaux..............................

Louise Hay, The class of recursively enumerable subsets of a recursively enumerable set ........................................

John Paul Helm, Albert Ronald da Silva Meyer and Paul Ruel Young, On orders of translations and enumerations...

Julien O. Hennefeld, A decomposition for $B(X)^{*}$ and unique Hahn-Banach

extensions

Gordon G. Johnson, Moment sequences in Hilbert space .

Thomas Rollin Kramer, A note on countably subparacompact spaces ...

Yves A. Lequain, Differential simplicity and extensions of a derivation ....

Peter Lorimer, A property of the groups Aut $\mathrm{PU}\left(3, q^{2}\right) \ldots$

225

Yasou Matsugu, The Levi problem for a product manifold.

231

John M.F. O'Connell, Real parts of uniform algebras ......

William Lindall Paschke, A factorable Banach algebra without bounded

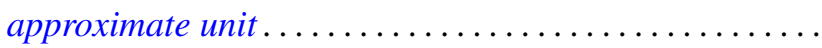

Ronald Joel Rudman, On the fundamental unit of a purely cubic field ....

Tsuan Wu Ting, Torsional rigidities in the elastic-plastic torsion of simply connected cylindrical bars .........................

Philip C. Tonne, Matrix representations for linear transformations on analytic sequences...................................

Jung-Hsien Tsai, On E-compact spaces and generalizations of perfect

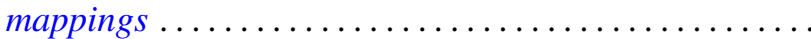

Alfons Van Daele, The upper envelope of invariant functionals majorized by an invariant weight. .. 